\title{
Influence of the 3D-2D crossover on the critical current of $\mathrm{Nb} / \mathrm{Cu}$ multilayers
}

\author{
Krasnov, V. M.; Pedersen, Niels Falsig; Oboznov, V. A.
}

Published in:

Physical Review B

Link to article, DOI:

10.1103/PhysRevB.50.1106

Publication date:

1994

Document Version

Publisher's PDF, also known as Version of record

Link back to DTU Orbit

Citation (APA):

Krasnov, V. M., Pedersen, N. F., \& Oboznov, V. A. (1994). Influence of the 3D-2D crossover on the critical current of Nb/Cu multilayers. Physical Review B, 50(2), 1106-1110. https://doi.org/10.1103/PhysRevB.50.1106

\section{General rights}

Copyright and moral rights for the publications made accessible in the public portal are retained by the authors and/or other copyright owners and it is a condition of accessing publications that users recognise and abide by the legal requirements associated with these rights.

- Users may download and print one copy of any publication from the public portal for the purpose of private study or research.

- You may not further distribute the material or use it for any profit-making activity or commercial gain

- You may freely distribute the URL identifying the publication in the public portal 


\title{
Influence of the 3D-2D crossover on the critical current of $\mathrm{Nb} / \mathrm{Cu}$ multilayers
}

\author{
V. M. Krasnov* and N. F. Pedersen \\ Physics Department, The Technical University of Denmark, DK-2800, Lyngby, Denmark
}

\author{
V. A. Oboznov \\ Institute of Solid State Physics, Russian Academy of Science, 142432 Chernogolovka, Moscow District, Russia
}

(Received 1 June 1993; revised manuscript received 28 March 1994)

\begin{abstract}
We report the experimental observation of the Josephson critical current across layers, $I_{c}^{\perp}$, for $\mathrm{Nb} / \mathrm{Cu}$ multilayers. Unique samples with a small cross section $(20 \mu \mathrm{m}$ in diameter) consisting of ten $\mathrm{Nb} / \mathrm{Cu}$ junctions were fabricated for such measurements. A strong influence of the dimensional 3D-2D crossover on the $I_{c}^{\perp}$ was observed. Thus, as the temperature becomes smaller than $T_{2 \mathrm{D}}$, hysteresis in the current-voltage characteristic appears and the behavior of the temperature dependence of the $I_{c}^{\perp}$ changes. For $T>T_{2 \mathrm{D}}$ the diminishing of the hysteresis is caused by a sharp decrease of the junction capacitance in the 3D regime when the sample becomes uniform across layers. Calculation of the criticalcurrent temperature dependence $I_{c}^{\perp}(T)$ for our multilayers was made. An agreement between experimental and theoretical dependencies $I_{c}^{\perp}(T)$ was found. From the theoretical simulations, we have obtained the dependence of the crossover and the critical temperatures of multilayers on the layer thicknesses, the boundary transparency, and layer conductivity.
\end{abstract}

\section{INTRODUCTION}

The properties of layered superconductors are of great interest nowadays particularly due to the discovery of high-temperature superconductivity (HTSC). There exist some experimental results ${ }^{1}$ showing the influence of the layered structure on the properties of HTSC. Yet usually it is difficult to interpret such HTSC data because of the complicated and often defective structures of HTSC samples. Investigation of the properties of pure multilayered structures such as artificial multilayers is then useful for the understanding of HTSC phenomena.

For the direct study of Josephson properties of multilayers, measurements with current applied across layers are of great interest. However, due to difficulties in the fabrication of a multilayered sample for such measurements there is a lack of experiments for this geometry. One of the main problems here is a very small transverse resistance of a thin metallic multilayered film. To increase it one should either increase the number of layers or decrease the area of the sample. However, in practice it is impossible to increase infinitely the number of layers, since the discrepancy in the layer parameters increases rapidly with the number of layers. On the other hand the progress in microfabrication technique makes it possible to fabricate samples with a very small cross-section area. ${ }^{2,3}$ Another possibility to increase a multilayer resistance is to use highly resistive tunnel superconductorinsulator-superconductor (SIS) multilayers. Recently a multilayered sample containing ten SIS (Nb/Al$\mathrm{AlO}_{x} / \mathrm{Nb}$ ) junctions was fabricated; ${ }^{4}$ however, the properties of the junctions were not very identical. Another feature of SIS multilayers is that the coupling between layers in such structures is always small. To study strong-coupling effects in superconducting multilayers such as a two-dimensional-three-dimensional (2D-3D) crossover we used a superconductor-normalmetal-superconductor (SNS) multilayered sample with the anisotropy of the lower critical field of the order of $10 .^{5}$

The 3D-2D crossover is one of the most interesting phenomena existing for layered superconductors. The dimensional crossover consists in the fact that at high temperatures, $T>T_{2 \mathrm{D}}$, (3D region) the multilayer behaves uniformly across layers. On the other hand at low temperatures, $T<T_{2 \mathrm{D}}$, (2D region) the multilayer behaves as a stack of distinct layers. Among the manifestations of the crossover that is intensively discussed now in the scientific literature is the depinning line observed for HTSC. ${ }^{6}$ This phenomenon is possibly caused by the disintegration of the straight vortex in the $2 \mathrm{D}$ region. Another well-studied manifestation of the dimensional crossover is the change in the $H_{c 2}$ temperature dependence at $T=T_{2 \mathrm{D}} \cdot{ }^{7,8}$ Recently other evidence for the 3D-2D crossover was observed in the $H_{c 1}$ temperature dependence of the $\mathrm{Nb} / \mathrm{Cu}$ multilayers. ${ }^{5}$ In that case a sharp increase of the anisotropy of the lower critical field was obtained at $T<T_{2 \mathrm{D}}$. Finally the direct observation of the process of subdivision of the multilayer on the individual junctions during the 3D-2D crossover was achieved in Ref. 9.

In this paper we study both experimentally and theoretically the influence of the 3D-2D crossover on the Josephson critical current of the multilayered bridges for current across layers. For this purpose multilayered $\mathrm{Nb} / \mathrm{Cu}$ samples with small area $\left(300 \mu \mathrm{m}^{2}\right)$ were fabricat- 
ed. Each sample consists of ten $\mathrm{Cu}$ layers with the thickness $d_{N}=150 \AA$ (sample I) and $200 \AA$ (sample II) and ten Nb layers with $d_{S}=200 \AA$ thus composing ten stacked SNS junctions. Our samples showed Josephson behavior, e.g., by applying RF power it was possible to observe Shapiro steps in the $I-V$ characteristics $(I V C)$ corresponding to all ten junctions. ${ }^{9}$

In this paper we have observed evidence for the 3D-2D crossover. It was found that the hysteresis of the IVC sharply decreases with increasing temperature. The characteristic temperature at which the hysteresis disappears is close to the $T_{2 \mathrm{D}}$ observed for the multilayers with the same parameters. ${ }^{5}$ This phenomenon is caused by the decrease of the junction capacitance in the $3 \mathrm{D}$ region when the sample becomes uniform across layers. Moreover the behavior of the critical current temperature dependence, $I_{c}^{\perp}(T)$, change at the crossover temperature.

The theoretical part of our paper consists of a calculation of the critical current temperature dependence for $S / N$ multilayers and in an investigation of dependencies of crossover and critical temperatures on the multilayer parameters.

Typically problems concerning the properties of layered superconductors are solved within the LawrenceDoniach (LD) model. ${ }^{10}$ The LD model yields for the 3D-2D crossover temperature

$$
\xi_{1}\left(T_{2 \mathrm{D}}\right)=s / \sqrt{2}
$$

where $\xi_{\perp}$ is the coherence length across layers and $s$ is the interlayer spacing. According to the LD model $H_{c 2}^{\|}$turns to infinity at this point. The LD model gives a rather crude approach especially for artificial multilayers in which layers are usually thick with respect to the coherence length. Another shortcoming of the LD model is that the interlayer coupling in this model is described by a temperature-independent phenomenological parameter. It was shown ${ }^{11-13}$ that for $S / N\left(S / S^{\prime}\right)$ multilayers this parameter depends on temperature (except for the case of very small transparencies of layers interfaces). In Refs. 12 and 13 for calculation of the proximity effect in SNS multilayers a method based on solving the GinzburgLandau (GL) equations across layers was used taking into account the exact boundary conditions at the interfaces. In this paper we use this method to calculate the Josephson critical current of our multilayers and to determine the temperature of the 3D-2D crossover, $T_{2 \mathrm{D}}$, in zero magnetic field. Our calculations show that $T_{2 \mathrm{D}}$ for SNS $\left(S S^{\prime} S\right)$ multilayers depends not only on the layer thicknesses but also on the particular parameters of layers such as boundary transparency $\beta$ and for the dirty case on the conductivity of the layers.

\section{EXPERIMENT}

The multilayer consists of ten $\mathrm{Cu}$ layers with the thickness $d_{N}=150 \AA$ (sample I) and $d_{N}=200 \AA$ (sample II) and ten $\mathrm{Nb}$ layers with the thickness $d_{S}=200 \AA$. The multilayered bridge was $20 \mu \mathrm{m}$ in diameter, made small to increase the sample resistance. Details of the sample fabrication may be found in Ref. 9.
The critical current at $4.2 \mathrm{~K}$ was about $230 \mathrm{~mA}$ for sample I and about $110 \mathrm{~mA}$ for sample II. Applying RF power with frequency $v$ at low temperatures we observed the fundamental Shapiro step at a voltage, $V_{10}=10(h / 2 e) v$, for both samples. ${ }^{9}$ This means that both samples really consist of ten SNS junctions in series, i.e., there were no shorts between layers. Moreover the properties of the ten junctions were close to each other. This could be judged first because the single resistive transition was observed for all ten junctions and second, since it was possible to synchronize all junctions simultaneously by a very small RF power.

In Fig. 1 the measured critical currents $I_{c}$ and the reverse currents $I_{r}$ for both $\mathrm{Nb} / \mathrm{Cu}$ multilayers are shown. ${ }^{9}$ The $I_{c}$ is the current at which the sample is switched from the superconducting to the resistive state when the current is increased and $I_{r}$ is the current at which the sample returns to the superconducting state when the current is decreased. The inset in Fig. 1 shows the resistive transition of sample II. Two superconducting transitions are seen, the first at $T \approx 7.8 \mathrm{~K}$ is that of the multilayer, the second at $T \approx 9 \mathrm{~K}$ is that of the $\mathrm{Nb}$ electrodes. Several common features are seen from Fig. 1. First, since the $\mathrm{Cu}$ layers are thicker for sample II, the critical current of this sample is smaller than that of sample I. Second, it is seen that at low temperatures there is a pronounced hysteresis in the $I V C$, while at high temperatures it disappears. Finally the slopes of $I_{c}(T)$ change at the point where the hysteresis disappears. Small current "tails" at high temperatures, $T>7.6 \mathrm{~K}$, are presumably caused by the transport of superconducting pairs from the thick electrodes through the multilayer, with the multilayer being in the normal state.

The hysteresis observed did not depend on the current

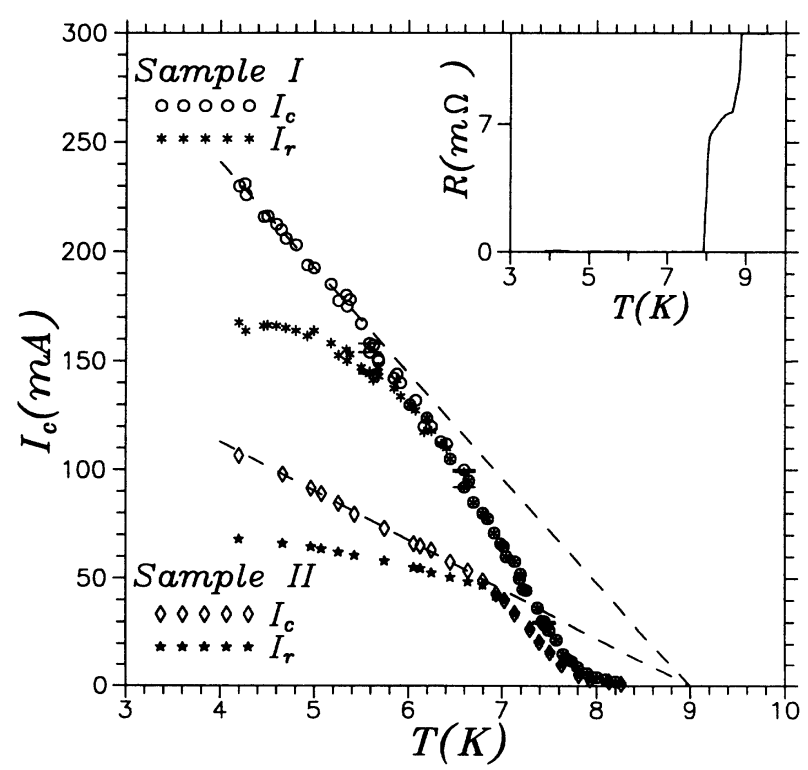

FIG. 1. Measured values of the critical current $I_{c}$ and the reverse current $I_{r}$ for sample $I$ and sample II. The inset shows the resistive transition of sample II. Two superconducting transitions are seen, the first at $T \approx 7.8 \mathrm{~K}$ is that of the multilayer, the second at $T \approx 9 \mathrm{~K}$ is that of the $\mathrm{Nb}$ electrodes. 
sweep rate and amplitude and it was not caused by sample heating. Typically the sample was cooled by the He gas. To prove that the hysteresis is not caused by the sample heating we immersed the sample in the liquid $\mathrm{He}$. Then we observed that the hysteresis even increased a little with respect to that in the He gas, since the real temperature of the sample becomes a little smaller due to the absence of the temperature gradient between the thermometer and the sample.

From Fig. 1 it is seen that, e.g., for sample I the hysteresis rapidly disappears at temperatures $5.5-6 \mathrm{~K}$. It is well known that the hysteretic behavior of the IVC is an intrinsic property of Josephson junctions. This phenomenon has been well studied, see, e.g., Ref. 14, and is caused by a finite capacitance $C$ of the junction. Approximately, if the McCumber parameter, $\beta_{c}=(2 e)$ ћ) $I_{c} R_{N}^{2} C$, is greater than unity, the $I V C$ is hysteretic and if $\beta_{c}<1$, then it is not. We may easily estimate the value of $\beta_{c}$ in the case when the multilayer is uniform across layers (i.e., in $3 D$ regime). Then the capacitance of the sample is equal to the capacitance of the $\mathrm{Nb}$ electrodes $C_{3 \mathrm{D}} \sim 1 \mathrm{pF}$ and $\beta_{c}^{3 \mathrm{D}}(4.2 \mathrm{~K}) \sim 0.02$. This estimation shows that at $T \sim 5.5 \mathrm{~K}$ the multilayer becomes nonuniform across layers and the capacitance of the junctions increases so that $\beta_{c}$ becomes of the order of unity. In other words, the $3 \mathrm{D}-2 \mathrm{D}$ crossover occurs at this temperature. Indeed the temperature of the crossover observed for such multilayers, $T_{2 \mathrm{D}}=5.5 \mathrm{~K},{ }^{5,7}$ corresponds to the observed temperature for the disappearance of hysteresis. The same behavior is seen for sample II. According to Eq. (1), $T_{2 \mathrm{D}}$ for sample II is larger than that for sample I. This result is in correspondence with the observed hysteresis behavior for sample II. Although it is difficult to estimate the capacitance of the junctions in the $2 \mathrm{D}$ regime, it is obvious that it is much larger than in the $3 \mathrm{D}$ region. The capacitance in the $2 \mathrm{D}$ state is caused by the finite transparency $\beta$ of the $\mathrm{Nb} / \mathrm{Cu}$ interface. Estimation of $\beta$ in our case gives $\beta=0.2-0.4 .^{15}$ The finite $\beta$ is also seen from the large transverse resistivity of the multilayers, $R_{n} \sim 7 \mathrm{~m} \Omega$, that is inversely proportional to the boundary transparency. ${ }^{16}$ Some additional mechanisms of intrinsic capacitance, e.g., caused by the finite relaxation time ${ }^{17}$ could also increase the total McCumber parameter of the junctions.

Simultaneously with the appearance of hysteresis at $T<T_{2 \mathrm{D}}$ the slope of $I_{c}(T)$ change. The dashed lines in Fig. 1 shows that in this region the behavior of $I_{c}$ for both samples is determined by the temperature $T=9 \mathrm{~K}$, which is different from the critical temperatures of the multilayers and approximately equal to the $T_{c}$ of the isolated $\mathrm{Nb}$ film.

\section{THEORY}

To understand the observed phenomena we performed a calculation of the proximity effect for our multilayers. We assumed that both $S$ and $N$ layers are in the dirty limit. To obtain the GL order parameters in the $S$ and $N$ layers we solve the GL equations across layers

$$
\begin{aligned}
& -\xi_{S}^{2}(T) \psi_{S}^{\prime \prime}-\psi_{S}+\psi_{S}^{3}=0, \quad \psi_{S}^{\prime}\left(d_{S} / 2\right)=0, \\
& -\xi_{N}^{2} \psi_{N}^{\prime \prime}+\psi_{N}+\psi_{N}^{3}=0, \quad \psi_{N}^{\prime}\left(-d_{N} / 2\right)=0
\end{aligned}
$$

with conditions at the $S / N$ boundary: ${ }^{18,19}$

$$
\begin{aligned}
& \psi_{S}^{\prime} /\left.\psi_{S}\right|_{S / N}=\left[p^{-1} \frac{\sigma_{S}}{\sigma_{N}} \xi_{N} \operatorname{coth}\left(\frac{d_{N}}{2 \xi_{N}}\right)\right]^{-1}, \\
& \left.\sigma_{N} \psi_{N}^{\prime}\right|_{S / N}=\left.\sigma_{S} \psi_{S}^{\prime}\right|_{S / N} .
\end{aligned}
$$

Here $\left|\psi_{S, N}\right|=\left|\Psi / \Psi_{0}\right|_{S, N}$ is the normalized GL order parameter. The function $p$ is given by ${ }^{19}$

$p=\left\{\begin{array}{l}1 / 2\left[\Psi\left(1 / 2+1 / 2 \gamma_{B}\right)-\Psi(1 / 2)\right], \quad \gamma_{M} \ll 1+\gamma_{B}, \\ 1 /\left[\gamma_{B}+7 \zeta(3) / \pi^{2}\right], \quad \gamma_{M} \gg 1+\gamma_{B} .\end{array}\right.$

Here $\quad \gamma_{M}=\gamma\left(d_{N} / 2 \xi_{N}\right), \quad \gamma=\left(\sigma_{N} \xi_{S}\right) /\left(\sigma_{S} \xi_{N}\right)$, $\gamma_{B}=\gamma_{B N}\left(d_{N} / 2 \xi_{N}\right)$, and $\gamma_{B N}=R_{B} / \rho_{N} \xi_{N} ; R_{B}$ is the product of the boundary resistance with the interface area and $\xi_{S, N}=\left(\hbar D_{S, N} / 2 \pi k_{B} T\right)^{1 / 2}$. Further for the description of the multilayer we shall use temperatureindependent parameters $\gamma, \gamma_{M}^{*}=\gamma_{M}\left(T_{c}\right), \gamma_{B}^{*}=\gamma_{B}\left(T_{c}\right)$, $\gamma_{B N}^{*}=\gamma_{B N}\left(T_{c}\right)$, and $\xi_{S, N}^{*}=\xi_{S, N}\left(T_{c}\right)$. The details of solving Eqs. (2) - (4) may be seen in Ref. 12.

The critical current of the Josephson $S / N$ junction is given by ${ }^{13}$

$$
J_{c}=\left.\left(J_{0} / \kappa\right) \frac{d \gamma}{\xi_{S}^{*} \gamma_{B N}^{*}}\left(\psi_{N} \psi_{S}\right)\right|_{S / N}
$$

Here $J_{0}$ and $\kappa$ are the critical current and the GL parameter of the bulk superconductor.

In Fig. 2 the temperature dependence of the product

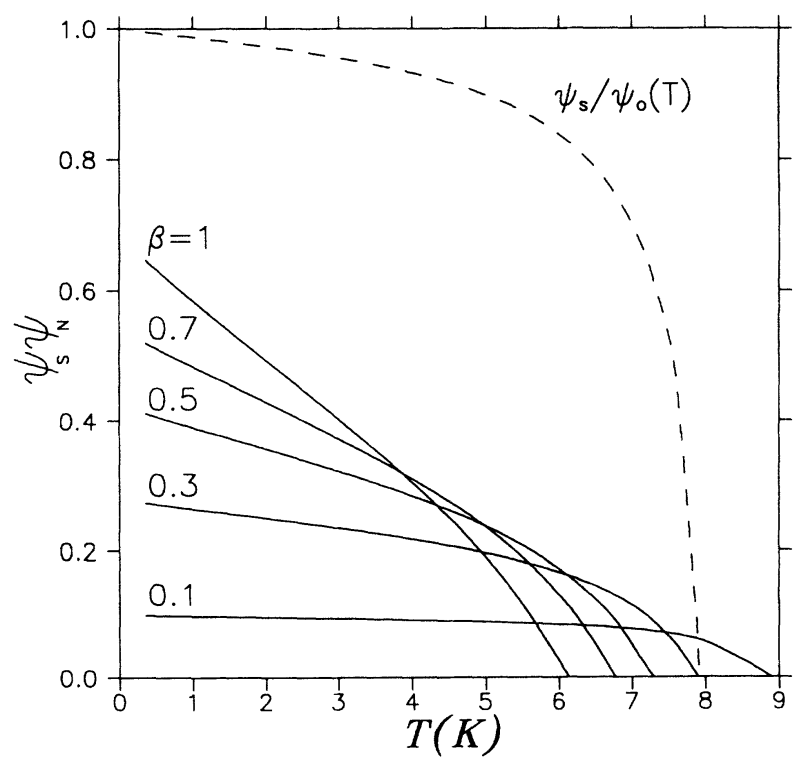

FIG. 2. The solid lines represent the calculated values of the product $\psi_{S} \psi_{N}$ proportional to $I_{c}$ for different values of the transparency $\beta$. The dashed line shows the temperature dependence of the normalized GL order parameter in $S$ layers for $\beta=0.3$. It is seen that the 3D-2D crossover occurs when $\psi_{S} / \psi_{0}(T) \sim 1$. The parameters chosen for the calculations were those corresponding to sample I. 
$\psi_{N} \psi_{S}$ for the different transparencies $\beta$ of the $S / N$ boundary, $[\beta \sim p$, Eq. (5)] are shown by solid lines. The parameters chosen for the calculations were those corresponding to sample I. To obtain the critical current scale the solid lines in Fig. 2 should be multiplied by the transparency factor $\beta$; it will then be seen that $I_{c}$ rapidly decreases with decreasing $\beta$ (approximately as $\beta^{2}$ ). The theoretical curves also shows that at some temperatures the slope of $I_{c}(T)$ changes showing the 3D-2D crossover. This transition is not sharp as in the LD model but rather smooth. From our calculation we can determine when the crossover occurs. The dashed line in Fig. 2 shows $\psi_{S}$ normalized to the bulk superconductor temperaturedependent GL order parameter $\psi_{0}(T)$ for $\beta=0.3$. It is seen that at temperatures below the crossover region $\psi_{S}$ becomes of the order of the equilibrium value $\psi_{0}(T)$. The pure 2D state of a multilayer is the case of isolated superconducting layers with $\psi_{S} / \psi_{0}(T)=1$. Thus it is natural to call the state of the multilayer at $T<T_{2 \mathrm{D}}$ when the order parameter of $S$ layers is nearly not perturbed, $\psi_{S} / \psi_{0}(T) \sim 1$, as the 2D state and it becomes clear why the experimentally observed critical current at $T<T_{2 \mathrm{D}}$ resembles the temperature dependence of $\mathrm{Nb}$ (see dashed lines in Fig. 1). The opposite state of the multilayer when $\psi_{S}$ is strongly suppressed corresponds to the $3 \mathrm{D}$ state when there is strong interaction between layers. Since the crossover from the 2D state to the 3D state is associated with the suppression of superconductivity, $T_{2 \mathrm{D}}$ is related to the critical temperature $T_{c}$ of the multilayer.

Taking the point where the slopes $J_{c}(T)$ and $\psi_{S}(T)$ change significantly as the crossover temperature we can obtain the dependence of $T_{2 \mathrm{D}}$ on the multilayer parameters. In Figs. 3(a) and 3(b) the calculated dependencies of $T_{2 \mathrm{D}}$ (solid lines) and $T_{c}$ of $S / N$ multilayers (dashed lines) are shown for different thicknesses of $S$ and $N$ layers. Other parameters are those estimated for our $\mathrm{Nb} / \mathrm{Cu}$ multilayers: $\beta=0.3, \sigma_{N} / \sigma_{S}=1.3$. It is seen from Fig. 3(b) that $T_{c}$ is strongly suppressed when $d_{S} / \xi_{S}^{*}<2$. The obtained $T_{c}\left(d_{S}\right)$ dependencies for $S / N$ multilayers are in qualitative agreement with the previous results for the SNS sandwiches. ${ }^{18,20}$ From Fig. 3 it is seen that both $T_{2 \mathrm{D}}$ and $T_{c}$ have the same tendency, however, there is a certain difference between them. In Fig. 4 the calculated dependencies of $T_{2 \mathrm{D}}$ and $T_{c}$ are shown for different transparencies $\beta$ with other parameters corresponding to sample I: $d_{S}=2.1 \xi_{S}^{*}, d_{N}=1.5 \xi_{N}^{*}, \sigma_{N} / \sigma_{S}=1.3$. In Fig. 5 the calculated dependencies of $T_{2 \mathrm{D}}$ and $T_{c}$ are shown for different ratios $\sigma_{N} / \sigma_{S}$, and constant $d_{S}=2 \xi_{S}^{*}, d_{N}=2 \xi_{N}^{*}$, $\beta=0.3$.

From Figs. 3-5 it is seen that $T_{2 \mathrm{D}}$ decreases when the transparency $\beta$ increases and in accordance with results obtained in Ref. $8 T_{2 \mathrm{D}}$ decreases when the values $\sigma_{N} / \sigma_{S}$ and $d_{N} \xi_{S} / d_{S} \xi_{N}$ are increasing. Thus the equation for $T_{2 D}$ is not so simple as that given by the LD model, Eq. (1). We emphasize that the reduction of $T_{2 D}$ is closely related with the reduction of the critical temperature $T_{c}$ of the multilayer.

We should mention that the 3D-2D crossover cannot be observed for arbitrary parameters of the multilayers. There exist at least two cases when this crossover is not seen in experiment: (i) $d_{S} / \xi_{S}^{*} \gg 1, d_{N} / \xi_{N}^{*} \gg 1, \beta \ll 1$, $\sigma_{N} / \sigma_{S} \ll 1$. Layers are decoupled and the multilayer is in the 2D state at all temperatures, i.e., $T_{2 \mathrm{D}} \sim T_{c}$. (ii) $d_{S} / \xi_{S}^{*} \ll 1, \beta \sim 1, \sigma_{N} / \sigma_{S}>1$. This is the case of very strong coupling between layers. The state of the multilayers is referred to as $2 \mathrm{D}$ strong coupling. ${ }^{5,7}$ It is seen from Fig. 2, the curve with $\beta=1$, that in this state there are hardly any peculiarities on the $I_{c}(T)$ dependence.
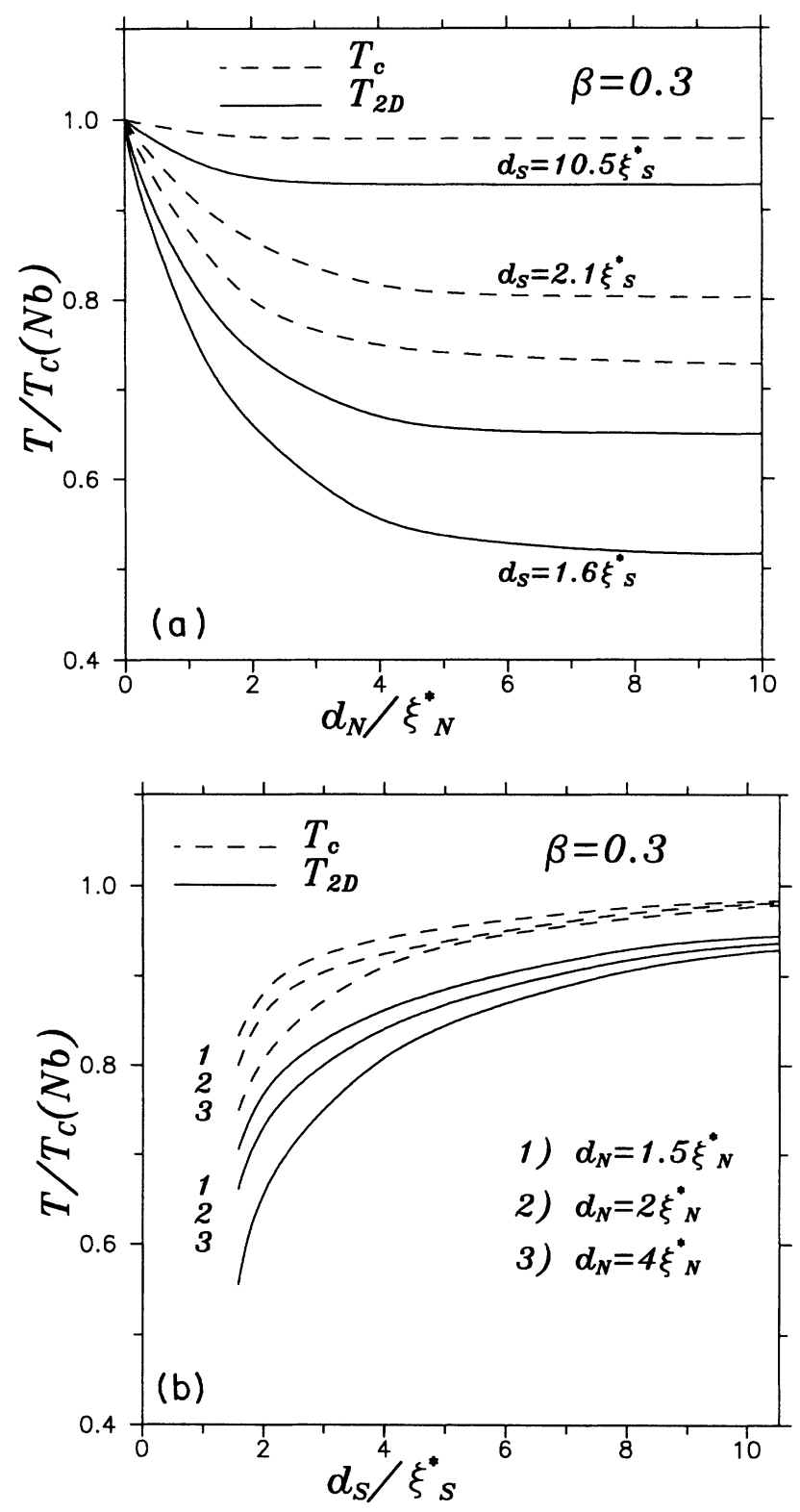

FIG. 3. (a) Calculated dependencies of $T_{2 \mathrm{D}}$ (solid lines) and $T_{c}$ (dashed lines) of $S / N$ multilayers for different thicknesses of $S$ layers as a function of the thickness of $N$ layers. Parameters used for calculations are those estimated for our $\mathrm{Nb} / \mathrm{Cu}$ multilayers: $\beta=0.3, \sigma_{N} / \sigma_{S}=1.3$. (b) Calculated dependencies of $T_{2 \mathrm{D}}$ (solid lines) and $T_{c}$ (dashed lines) of $S / N$ multilayers for different thicknesses of $N$ layers as a function of the thickness of $S$ layers. Parameters used for calculations are the same as in Fig. 3(a). It is seen that $T_{c}$ is strongly suppressed when $d_{s} / \xi_{S}^{*}<2$. 


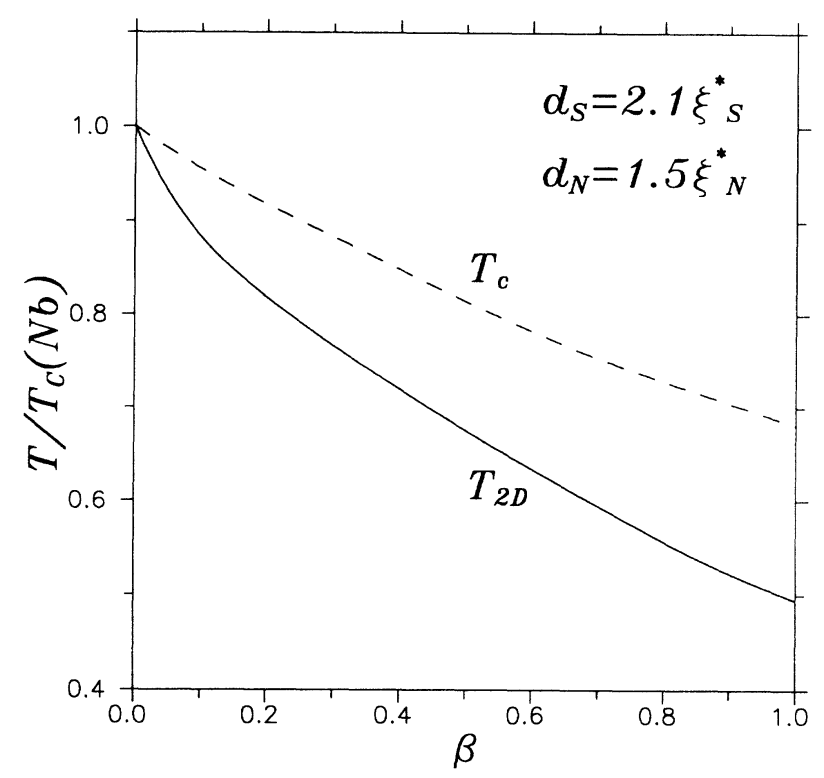

FIG. 4. Calculated dependencies of $T_{2 \mathrm{D}}$ (solid lines) and $T_{c}$ (dashed lines) as a function of transparency $\beta$. Parameters of calculation are those for sample $\mathrm{I}: d_{S}=2.1 \xi_{S}^{*}, d_{N}=1.5 \xi_{N}^{*}$, $\sigma_{N} / \sigma_{S}=1.3$.

\section{CONCLUSIONS}

In conclusion we have studied both experimentally and theoretically the influence of the 3D-2D crossover on the Josephson critical current of $\mathrm{Nb} / \mathrm{Cu}$ multilayers. For this purpose the unique sample consisting of ten SNS junctions was fabricated. New evidence for the dimensional 3D-2D crossover was observed: (i) at $T>T_{2 \mathrm{D}}$ the hysteresis of the $I V C$ disappears and (ii) the slope of $I_{c}(T)$ change at $T \sim T_{2 \mathrm{D}}$. The disappearance of the hysteresis is caused by the decrease of the junction capacitance in the 3D state. The calculations show that the crossover occurs at $T_{2 \mathrm{D}}$ when $\psi_{S} / \psi_{0}\left(T_{2 \mathrm{D}}\right) \sim 1$. Thus in the 2D region the temperature dependence of $I_{c}$ resembles the

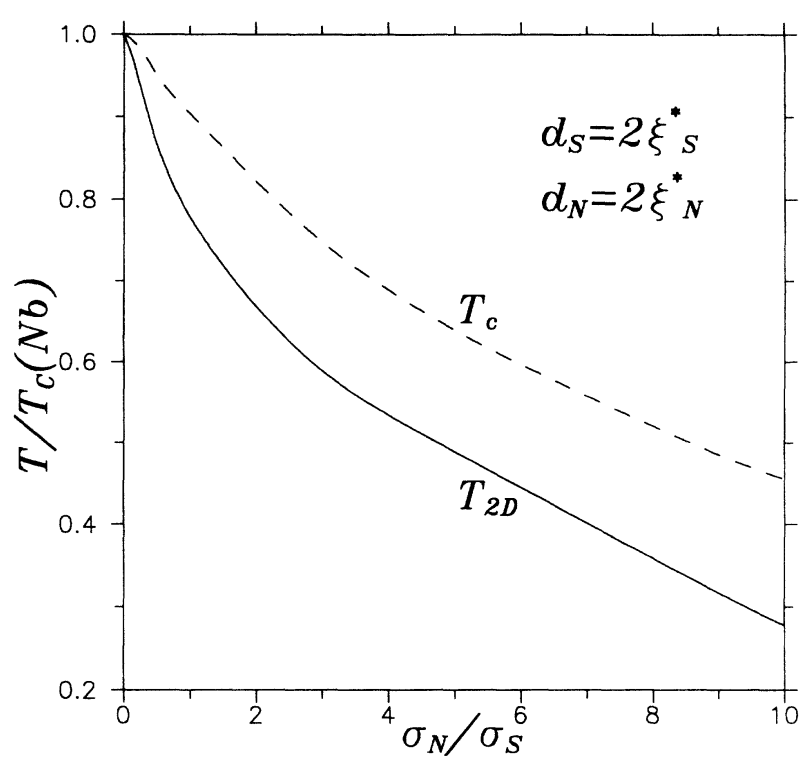

FIG. 5. Calculated dependencies of $T_{2 \mathrm{D}}$ (solid lines) and $T_{c}$ (dashed lines) as a function of the ratio $\sigma_{N} / \sigma_{S}$. Parameters of calculation are $d_{S}=2 \xi_{S}^{*}, d_{N}=2 \xi_{N}^{*}, \beta=0.3$.

behavior of the isolated superconductor. $T_{2 \mathrm{D}}$ decreases when the transparency $\beta$ and the values $\sigma_{N} / \sigma_{S}$ and $d_{N} \xi_{S} / d_{S} \xi_{N}$ are increasing without a simple relation, e.g., Eq. (1), given by the LD model. The reduction of $T_{2 \mathrm{D}}$ is closely related to the reduction of the critical temperature $T_{c}$ of the multilayer.

\section{ACKNOWLEDGMENTS}

The authors are grateful to J. Mygind for helpful assistance in the experimental setup and to A. Golubov for helpful discussions. The support from NATO Linkage Grant No. LG 9206972 is gratefully acknowledged.
*Permanent address: Institute of Solid State Physics, Russian Academy of Science, 142432 Chernogolovka, Moscow District, Russia.

${ }^{1}$ R. Kleiner, F. Steinmeyer, G. Kunkel, and P. Muller, Phys. Rev. Lett. 68, 2394 (1992).

${ }^{2}$ M. A. M. Gijs, S. K. J. Lenczowski, and J. B. Giesbers, Phys. Rev. Lett. 70, 3343 (1993).

${ }^{3}$ R. J. Highmore, M. G. Blamire, R. E. Somekh, and J. E. Evetts, J. Magn. Magn. Mater. 104, 1777 (1992).

${ }^{4}$ H. Kohlstedt, G. Hallmanns, I. Nevirkovets, G. Guggi, and C. Heiden, IEEE Trans. Appl. Supercond. 3, 2197 (1993).

${ }^{5}$ V. M. Krasnov, A. E. Kovalev, V. A. Oboznov, and V. V. Ryazanov, Physica C 215, 265 (1993).

${ }^{6}$ L. I. Glazman and A. E. Koshelev, Phys. Rev. B 43, 2835 (1991).

${ }^{7}$ I. Banerjee ard I. Schuller, J. Low Temp. Phys. 54, 501 (1984).

${ }^{8}$ S. Takahashi and M. Tachiki, Phys. Rev. B 33, 4620 (1986).

${ }^{9}$ V. M. Krasnov, N. F. Pedersen, V. A. Oboznov, and V. V. Ryazanov, Phys. Rev. B 49, 12969 (1994).
${ }^{10}$ W. E. Lawrence and S. Doniach, Proceedings of the 12th International Conference on Low Temperature Physics (Academic Press of Japan, Kyoto, 1971), p. 361.

${ }^{11}$ L. Bulaevskii and M. Zyskin, Phys. Rev. B 42, 10230 (1990).

${ }^{12}$ A. Golubov and V. Krasnov, Physica C 196, 177 (1992).

${ }^{13}$ V. M. Krasnov, N. F. Pedersen, and A. A. Golubov, Physica C 209, 579 (1993).

${ }^{14}$ D. E. McCumber, J. Appl. Phys. 39, 3113 (1968).

${ }^{15}$ V. M. Krasnov, V. A. Oboznov, and V. V. Ryazanov, Physica C 196, 335 (1992).

${ }^{16}$ M. Yu. Kuprianov and V. F. Lukichev, Zh. Eksp. Teor. Fiz. 94, 139 (1988) [Sov. Phys. JETP 66, 1163 (1988)].

${ }^{17}$ X. Song, J. Appl. Phys. 47, 2651 (1976).

${ }^{18}$ P. G. De Gennes, Rev. Mod. Phys. 36, 225 (1964).

${ }^{19}$ A. A. Golubov and M. Yu. Kupriyanov, Zh. Eksp. Teor. Fiz. 96, 1420 (1989) [Sov. Phys. JETP. 69, 805 (1989)].

${ }^{20}$ A. A. Golubov, M. Yu. Kupriyanov, V. F. Lukichev, and A. A. Orlikovskii, Sov. J. Microelectronics 12/4, 355 (1983). 